\title{
FT-IR INVESTIGATIONS OF BEDO-TTF AND RADICAL SALTS OF BEDO-TTF
}

\section{J. MOLDENHAUER, K. I. POKHODNIA, D. SCHWEITZER}

3. Physikalisches Institut, Universität Stuttgart, Pfaffenwaldring 57, 7000 Stuttgart 80 (Germany)

\section{HEINEN}

Max-Planck-Institut für Medizinische Forschung, AG: Molekülkristalle, Jahnstr. 29, 6900 Heidelberg (Germany)

\section{H. J. KELLER}

Anorganisch-Chemisches Institut, Universität Heidelberg, Im Newenheimer Feld 270, 6900 Heidelberg (Germany)

\begin{abstract}
We investigated powder absorption spectra of the donor BEDO-TTF and some radical salts of BEDO-TTF, which are metals even at low temperatures. After an assignment of the bands in the neutral donor we found in the radical salts different vibrational frequencies of bands correlated with $\mathrm{C}-\mathrm{O}$ vibrations. These frequencies are directly related to the average charge on the donor molecule. Additionally frequencies of $\mathrm{CH}_{2}$-stretching vibrations exhibit differences, which can be ascribed to a varying strength of donor-anion interaction depending on the respective anion.
\end{abstract}

\section{Introduction}

The successful history of radical cation salts of the organic donor BEDT-TTF (bis(ethylenedithiolo)tetrathiafulvalene) concerning metallic conductivity and superconductivity with $\mathrm{T}_{\mathrm{c}}$ up to $12.5 \mathrm{~K}$ [1] has always produced attempts towards modifying this donor. One modification consists in the substitution of the outer sulfur atoms through oxygen, yielding BEDO-TTF (bis(ethylendioxy)tetrathiafulvalen) (Fig.1), which was synthesized for the first time by Suzuki et al. [2]. The aim in this substitution was twofold [3] : Firstly BEDO-TTF as a constituent in a radical cation salt now contains lighter atoms than BEDT-TTF, therefore raising $\mathrm{T}_{\mathrm{c}}$ according to BCS-theory, and secondly a smaller conduction bandwidth was

\footnotetext{
* On leave from Institute of Semiconductors, Ukrainian Academy of Sciences, Kiev, Ukrainia
} 
expected, which should increase the density of states near the Fermi level. In the meantime a lot of radical cation salts have been synthesized, two of them exhibiting ambient-pressure superconductivity - (BEDO-TTF $)_{3} \mathrm{Cu}_{2}(\mathrm{NCS})_{3}[4]$ with $\mathrm{T}_{\mathrm{c}}=1.06 \mathrm{~K}$ and $(\mathrm{BEDO}-\mathrm{TTF})_{2} \mathrm{ReO}_{4} \cdot \mathrm{H}_{2} \mathrm{O}$ [5] with an onset of superconductivity at $2.5 \mathrm{~K}$.

Fig. 1.

The BEDO-TTF molecule<smiles>[H][R]1([H])OC2SC(=C3SC4OC5OC(O4)C(O5)S3)SC2O1</smiles>

The aim of the spectroscopic work on the neutral donor BEDO-TTF and a few radical cation salts of it was an assignment of the fundamental vibrations of BEDO-TTF according to the assignment of BEDT-TTF (recently a normal coordinate analysis has been carried out by Pokhodnia et al. [6]), an investigation of the dependence of vibrational frequencies on the average charge per donor molecule and of the behaviour of the $\mathrm{CH}_{2}$-stretching frequencies in different salts.

\section{Experimental}

The donor BEDO-TTF was synthesized as described by Suzuki et al. [2], the crystals of the radical cation salts investigated in this work have been prepared electrochemically using the crown ether route [7]. For infrared absorption measurements, $0.8 \mathrm{mg}$ of crystals were ground with $200 \mathrm{mg} \mathrm{KBr}$ in an agate mortar and afterwards pressed to pellets with a pressure of $9 \mathrm{t} / \mathrm{cm}^{2}$.

The spectra were taken on a commercial Fourier transform spectrometer (BOMEM DA 3.02) with a fast InSb detector in the range of $5000-1800 \mathrm{~cm}^{-1}$ and a MCT detector between $1800 \mathrm{~cm}^{-1}$ and $450 \mathrm{~cm}^{-1}$ - the applied resolution was $0.5 \mathrm{~cm}^{-1}$. In order to obtain a good signal-to-noise ratio, 2000 interferograms were coadded for each spectrum.

\section{Results}

\section{A. The molecular vibrations of the donor BEDO-TTF.}

The vibrational bands of BEDO-TTF in the range between $450 \mathrm{~cm}^{-1}$ and $1750 \mathrm{~cm}^{-1}$ are shown in Fig. 2, the insert displays the $\mathrm{CH}_{2}$-stretching vibrations around $3000 \mathrm{~cm}^{-1}$. 


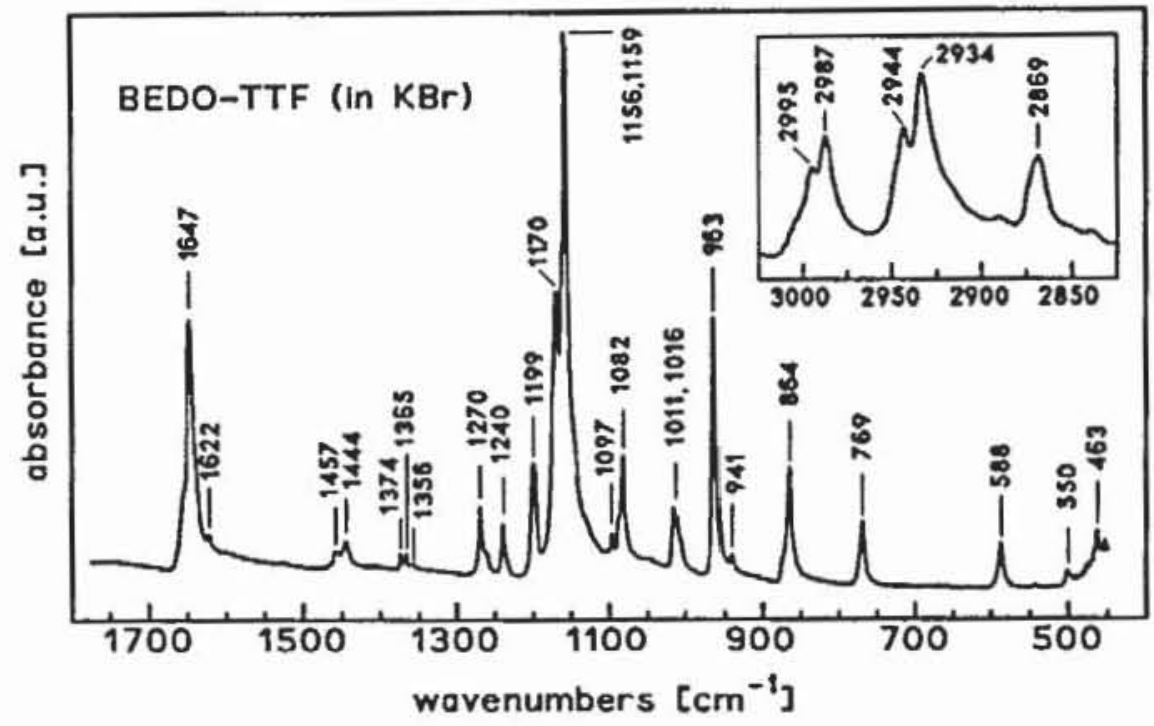

Fig. 2. Vibrational spectrum of BEDO$\mathrm{TTF}$ in $\mathrm{KBr}$

Comparing with the vibrations of BEDT-TTF [8], the normal coordinate analysis in [6] shows that the substitution of sulfur by oxygen in the outer rings leads to a strongly different distribution of the charge on the molecule as in the latter. Therefore many bands are expected to appear with different frequencies, especially when the C-O-C-groups are involved in the respective fundamental vibration. Apart from these vibrations we observe mainly two remarkable facts: First of all the $\mathrm{CH}_{2}$-groups are only slightly affected by this redistribution with respect to BEDT-TTF - the frequency shift of their stretching vibration (i.e. vibrations with no other bonds involved) is less than $1 \%$. On the other hand, there is a strong change in the vibrations of the $\mathrm{C}=\mathrm{C}$-bonds in the center and in the rings whereas the symmetric stretching of the central $\mathbf{C}=\mathrm{C}$-bond (Raman active) is only sligthly shifted upwards by $\approx 2 \%$, the shift of the in-phase as well as of the anti-phase stretching of the ring $\mathrm{C}=\mathrm{C}$ amounts $\approx 150 \mathrm{~cm}^{-1}$ or nearly $10 \%$.

This fact should strongly affect the frequencies of the appearing vibronic band in the radical salts of BEDO-TTF, which in the observed frequency range are caused by the latter mentioned in-phase vibration inducing a coupling of the radical electrons to this vibration.

\section{B. The molecular vibrations of radical cation salts (BEDO-TTF) $)_{\mathbf{m}} \mathbf{X}_{\mathbf{n}}$}

Aside from the above mentioned salts with superconducting phase transitions $(\mathrm{BEDO}-\mathrm{TTF})_{3} \mathrm{Cu}_{2}(\mathrm{NCS})_{3}$ and $(\mathrm{BEDO}-\mathrm{TTF})_{2} \mathrm{ReO}_{4} \cdot \mathrm{H}_{2} \mathrm{O}$ we investigated as well (BEDO-TTF $)_{2.4} \mathrm{I}_{3}[3]$ and a BEDO-TTF chloride phase, which both exhibit metallic behaviour. The exact composition of the investigated BEDO-TTF chloride phase is not known because of the poor crystal quality. This phase is designated as "(BEDO-TTF $)_{2} \mathrm{Cl}^{\prime}$ in Figs. 3 and 4 because of the IR data dicussed below. 
The absorption spectra of different radical salt of BEDO-TTF between 1800 and $600 \mathrm{~cm}^{-1}$ are shown in Fig. 3. The overall structure is the same for all spectra

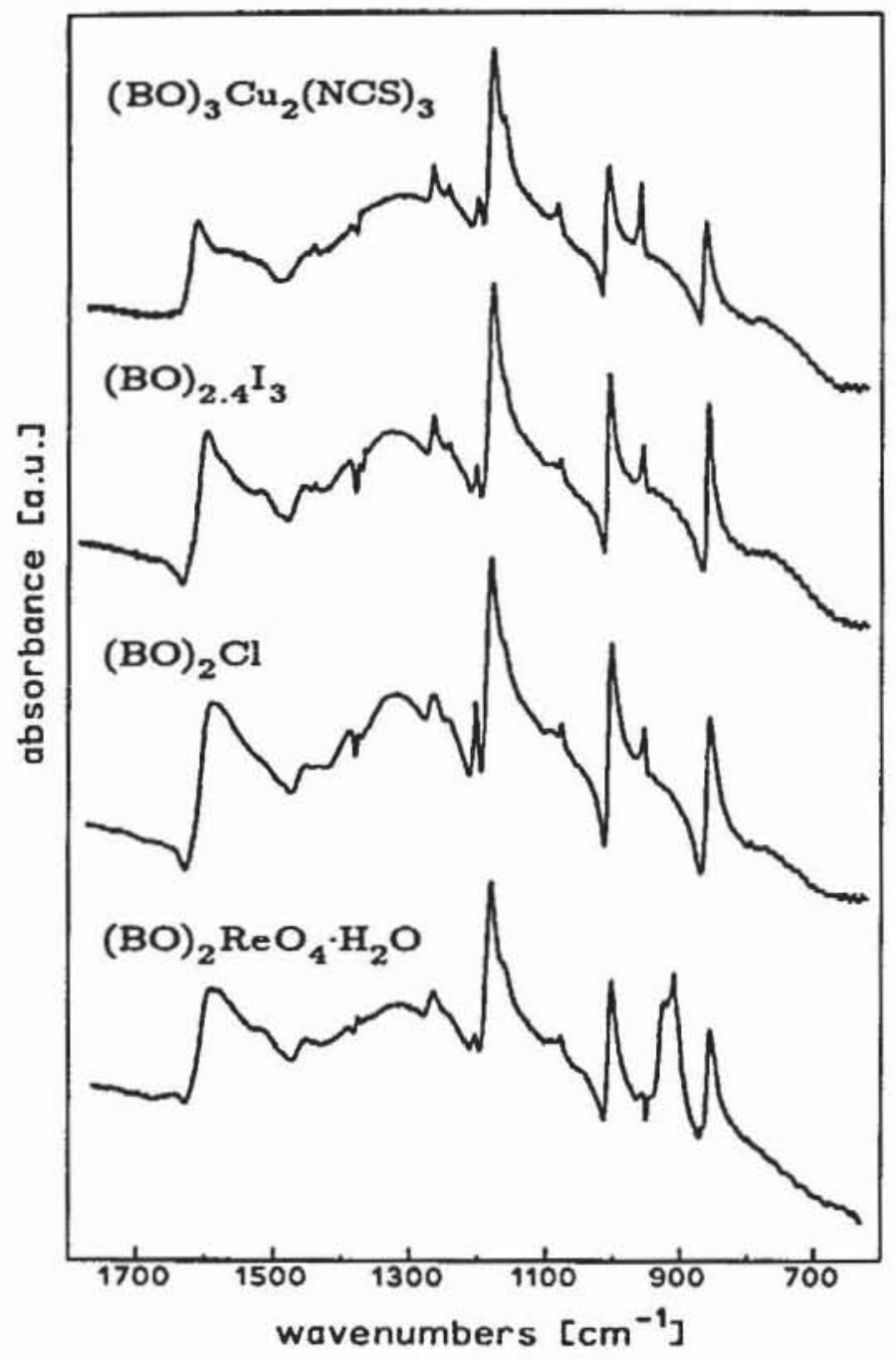

Fig. 3. IR absorption spectra of radical cation salts of BEDO-TTF (abbreviated as BO) (except for the presence of a band at $910 \mathrm{~cm}^{-1}$ in the $\mathrm{ReO}_{4}$-salt, which is due to a vibration of the anion) - the prominent features are the broad bands around 1600 $\mathrm{cm}^{-1}$, and a few remarkably strong bands between 1200 and $800 \mathrm{~cm}^{-1}$. According to the normal coordinate analysis for the neutral BEDOTTF [6], most of the latter are due to vibrations including $\mathrm{C}-\mathrm{O}$ bonds. The assignments presented there as well give an explanation for the origin of the broad bands mentioned before: They have to be taken as vibronic bands due to EMV coupling, as this was already discussed [9] for BEDT-TTF salts.

A closer examination of the frequencies of all these mentioned bands is given in the subsequent discussion.

\section{C. $\mathrm{CH}_{2}$-stretching vibrations}

Concerning the above mentioned different average charge on the donor molecules, ranging from +0.33 to +0.5 in the different investigated salts, it could be shown [10] in radical salts of BEDT-TTF, that the frequencies of the $\mathrm{CH}_{2}$-stretching vibrations are nearly unaffected by the respective charge per donor molecule. A comparison with those of BEDO-TTF shows, that a substitution in the outer rings as in the present case causes a shift to higher frequencies of nearly $1 \%$, but the behaviour after ionization is exspected to be the same as in BEDT-TTF. The shifts observed in these radical salts are rather due to the donor-acceptor interactions, which are mediated by the terminal ethylene groups, as long as the anions are lying in sheets separating the donor stacks. 


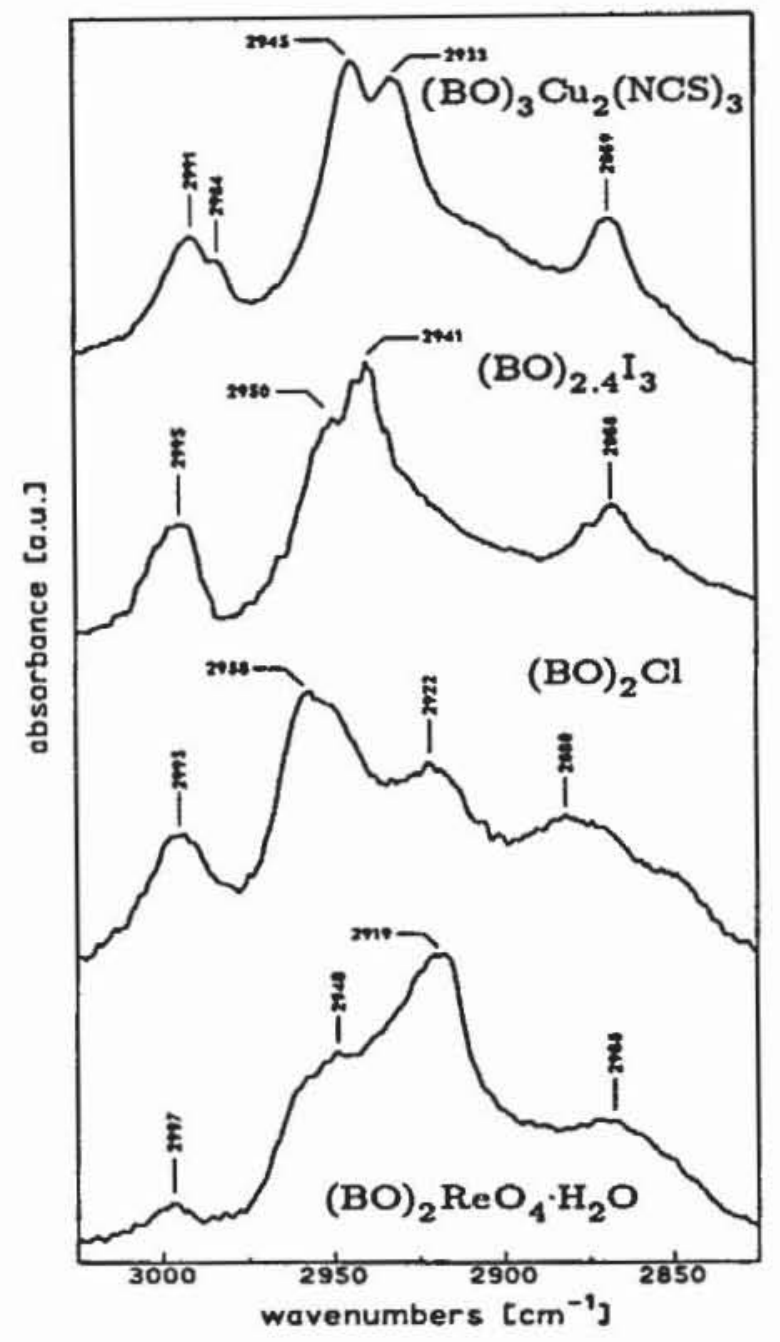

In turning our attention to the $\mathrm{CH}_{2}$-stretching vibrations in radical salts of BEDO-TTF, which are shown in Fig. 4 (a baseline correction has been applied), we first of all should compare the observed bands with those found in neutral BEDO-TTF. In (BEDO-TTF) $\mathrm{Cu}_{2}(\mathrm{NCS})_{3}$ the observed differences are very small, increasing on going from the iodine salt to the $2: 1$ salts. Refering to the situation found in BEDT-TTF it is worth noting, that in neutral BEDOTTF the interaction between the molecules is determined by short $\mathrm{C}-\mathrm{H} \cdots \mathrm{O}$ contacts [11] and it is reported - at least for $(\mathrm{BEDO}-\mathrm{TTF})_{3} \mathrm{Cu}_{2}(\mathrm{NCS})_{3}$ [4] and the iodine salt [3], that these contacts are dominating the packing motifs even of the radical salts.

Fig. 4. $\mathrm{CH}_{2}$-stretching bands of BEDO-TTF (abbreviated as BO) radical salts

\section{Conclusions}

The $\mathrm{CH}_{2}$-stretching vibrations therefore indicate an increasing interaction on going from an anion with weaker or more diluted electronegative constituents like $\mathrm{Cu}_{2}(\mathrm{NCS})_{3}^{-}$to anions with a higher electron affinity like $\mathrm{Cl}^{-}$and $\mathrm{ReO}_{4}^{-}$. But a stronger interaction of $\mathrm{CH}_{2}$-groups with electronegative anions like the latter means presence of an at least weak hydrogen bonding, which should decrease the frequency of $\mathrm{CH}_{2}$-stretching vibrations. Concerning the most intensive bands we observe both a shift to higher frequencies as well as to lower. Therefore we propose, that the strong donor-donor interaction via $\mathrm{C}-\mathrm{H} \cdots \mathrm{O}$ contacts (as known from neutral BEDO-TTF) is a strong competitor for donor-anion contacts as long as the electronegativity of the anions is small. This might be as well the reason for the fact that in the iodide-salt and (BEDO-TTF) ${ }_{3} \mathrm{Cu}_{2}(\mathrm{NCS})_{3}$ an incommensurable sublattice and a commensurable superlattice respectively is found.

On the other hand, donor-donor interactions are surely influenced by the average charge on the donor. Significant dependencies of vibrational frequencies of several fundamental modes are given in Fig. 5 - it should be noted, that all these modes involve vibrations of $\mathrm{C}-\mathrm{O}$ bonds. From the decrease in frequencies it is 


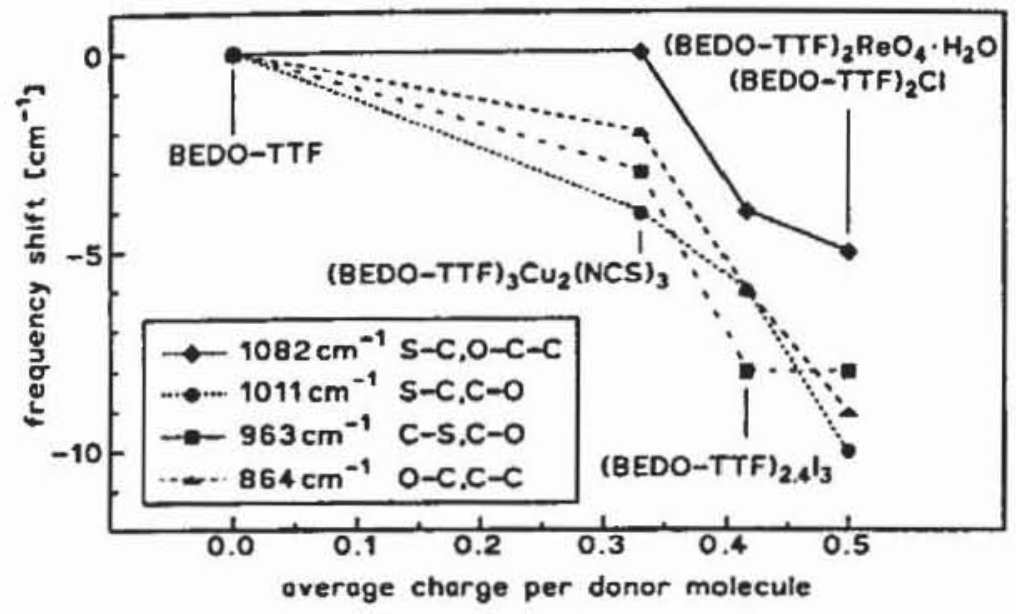

Fig. 5.

Dependence of frequency shifts of several vibrational modes (with respect to BEDO-TTF) on the average charge per donor molecule. The applied resolution was $0.5 \mathrm{~cm}^{-1}$.

clear, that the transferred charge mainly stems from the $\pi$-electron framework contributed by oxygen atoms. The fact that the observed shifts of (BEDO-TTF) ${ }_{2}$ $\mathrm{ReO}_{4} \cdot \mathrm{H}_{2} \mathrm{O}$ and of the investiged BEDO-TTF chloride salt are identical supports the assumption of an identical stoichiometry for the chloride phase.

Additionally some information about the donor-donor interaction can be extracted from the positions of the vibronic band, which range from $1610 \mathrm{~cm}^{-1}$ to $1585 \mathrm{~cm}^{-1}$. But for a reliable interpretation the Raman spectra of the radical salts in question are necessary, which are not available yet.

Acknowledgements - We gratefully acknowledge financial support of this work by the Forschungsschwerpunkt Supraleiter des Landes Baden-Württemberg. K.I.P. would like to thank for a grant of the DAAD.

\section{References}

[1] A.M.Kini, U.Geiser, H.H.Wang, K.D.Carlson, J.M.Williams, H.K.Kwok, K.G.Vandervoort, J.E.Thompson, D.L.Stupka, D.Jung, M.H.Whangbo; Inorg. Chem. 29 (1990), 2555

[2] T.Suzuki, H.Yamochi, G.Srdanov, K.Hinkelman, F.Wudl; J. Am. Chem. Soc. 111 (1989), 3108

[3] F.Wudl, H.Yamochi, T.Suzuki, H.Isotalo, C.Fite, H.Kasmai, K.Liou, G.Srdanov, P.Coppens, K.Maly, A.Frost-Jensen; J. Am. Chem. Soc. 112 (1990), 2 \&61

[4] M.A.Beno, H.H.Wang, A.M.Kini, K.D.Carlson, U.Geiser, W.K.Kwok, J.E.Thompson, J.M.Williams, J.Ren, M.H.Whangbo; Inorg. Chem. 29 (1990), 1599

[5] S.Kahlich, D.Schweitzer, I.Heinen, Song En Lan, B.Nuber, H.J.Keller, K.Winzer, H.W.Helberg; Solid State Comm. 80, No.3 (1991), 191

[6] K.I.Pokhodnia, M.E.Kozlov, V.G.Onischenko, D.Schweitzer, J.Moldenhauer, R.Zamboni; these Proceedings

[7] U.Geiser, H.H.Wang, K.M.Donega, B.A.Anderson, J.M.Williams, J.F.Kwak; Inorg. Chem. 25 (1986), 401

[8] M.E.Kozlov, K.I.Pokhodnia, A.A.Yurchenko; Spectrochim. Acta 43A, No.3 (1987), 323

[9] J.Moldenhauer, K.I.Pokhodnia, D.Schweitzer, H.J.Keller; these Proceedings

[10] J.Moldenhauer, D.Schweitzer, H.J.Keller; To be published

[11] J.J.Novoa, M.H.Whangbo, J.M.Williams; Mol. Cryst. Liq. Cryst. 181 (1990), 25 